\title{
Growing a Peer Review Culture among Graduate Students
}

\author{
Vinícius Medina Kern ${ }^{1,2}$, Osmar Possamai ${ }^{1}$, Paulo Mauricio Selig ${ }^{1}$, \\ Roberto Carlos dos Santos Pacheco ${ }^{1}$, Gilberto Corrêa de Souza ${ }^{1}$, Sandro Rautenberg ${ }^{1,3}$, \\ and Renata Tavares da Silva Lemos ${ }^{1}$ \\ ${ }^{1}$ Universidade Federal de Santa Catarina (UFSC), Programa de Pós-Graduação em Engenharia \\ e Gestão do Conhecimento (EGC), Brasil \\ \{kern, possamai, selig, pacheco, gilberto, srautenberg\} @egc.ufsc.br, \\ renata.lemoz@eletrocooperativa.org \\ www. egc.ufsc.br \\ ${ }^{2}$ Instituto Stela, Brasil \\ www.stela.org.br \\ ${ }^{3}$ Universidade Estadual do Centro-Oeste (Unicentro), Brasil \\ www. unicentro.br
}

\begin{abstract}
Usual processes for pursuing education excellence in a graduate ${ }^{1}$ program are candidate selection, coursework, research, and thesis defense. This paper is an experience report on a complementary approach: the growing of a peer review culture among graduate students. We instruct first-year masters and doctoral students on principles for preparing a thesis proposal. Students present their proposals in collective discussion sessions with feedback from professors. The students then submit their proposals through a web interface and are instructed on the role they will play next - of anonymous referees of their peers' proposals. The referee reports and general statistics are made available to all participating students and advisers. Updated proposals are submitted to an annual workshop open to all participating students and advisers. About 60 students take part in this annual series of seminars with peer review and workshop, generating individual thesis proposals and 180 referee reports, 3 for each proposal. Students and their advisers receive detailed feedback on individual participation as author and referee. The main strength of this experience is the opportunity to assimilate the techniques of objective criticism and to reflect about the quality of own and others' work. The paper outlines future research and development issues.
\end{abstract}

Keywords: Research, Culture, Assessment, Knowledge Society, Higher Education.

\section{Introduction}

A graduate program is a school system that conducts research and educates new researchers - the graduate students. Common processes for pursuing education excellence are candidate selection, teaching and coursework, research (conducted by

\footnotetext{
${ }^{1}$ We use the North-American terminology 'graduate', the same as the British 'postgraduate'.
} 
students under supervision), and thesis defense. Those processes require individual efforts from the students (such as in the writing of a proposal for candidate selection) and also establish vertical relationships (as in advising and defending in front of a committee) and horizontal relationships (as in student teaming for coursework). In this paper, we introduce our approach to a complementary process aimed at the collective level: the growing of a peer review culture among graduate students.

The research object of our Graduate Program in Knowledge Engineering and Management (KEM), started in 2004, is "knowledge as a production factor", 2 KEM confirms the predictions by Angelov, Melnik, and Buur [1] that "an increasing number of very strong students will look for a multidisciplinary education": our annual admission has attracted between 322 and 423 candidates for about 60 noscholarship openings, since 2004.

Given the intrinsic interdisciplinary character of the research object, our program runs the risk, as pointed out by Bunge [2], of being "multidisciplinary, hence potentially dispersive, rather than interdisciplinary, hence cohesive". Interdisciplinarity requires excellent communication.

The need for enhancing communication was one of the motivations for our first annual workshop in 2004, after only 6 months of existence, in which the students were asked to present their thesis proposals. From 2005 on, we established research seminars in preparation for the workshop, including the peer review process discussed in this paper.

In the next sections, we analyze our graduate education system under the systemism of Mario Bunge and we give a concise account of the method used for the method used for the peer review of thesis proposals. Results of this approach are presented. Finally, we discuss the outcomes and open research and development issues, including instrumental ones, for instance automating some knowledgeintensive tasks, and also methodological issues such as connecting the seminars with a new mandatory course on the scientific method in order to strengthen students' preparedness to provide objective, professional criticism.

\section{A Systemic View of Graduate Learning}

According to a systemic worldview [3], "systems have systemic (emergent) features that their components lack" and everything is a system or an actual or potential component of a system. We outline our graduate learning environment using the CESM model [2], according to which any concrete system $\sigma$ can be modeled as $\mu(\sigma)$ $=\langle\mathrm{C}(\sigma), \mathrm{E}(\sigma), \mathrm{S}(\sigma), \mathrm{M}(\sigma)>$, i.e., the list of the system's composition, environment, structure (bonds among components and between those and items of the environment), and mechanism (the process(es) that makes the system tick).

Figure 1 illustrates our model of graduate learning system: the components (inside the ellipse) are students (S) and professors (P). The environment includes (clockwise from upper right) the laws and regulations that affect the graduate program $(\mathrm{R})$, the academic community of the Brazilian graduate education system (A), the host university, its departments and staff (U), funding agencies (\$), other organizations $(\mathrm{O})$, and the culture in which the program is immersed (C).

\footnotetext{
${ }^{2}$ In: Interaction of the Program's research areas in search of the research object (in Portuguese), http://www.egc.ufsc.br/htms/vermais_index.htm
} 


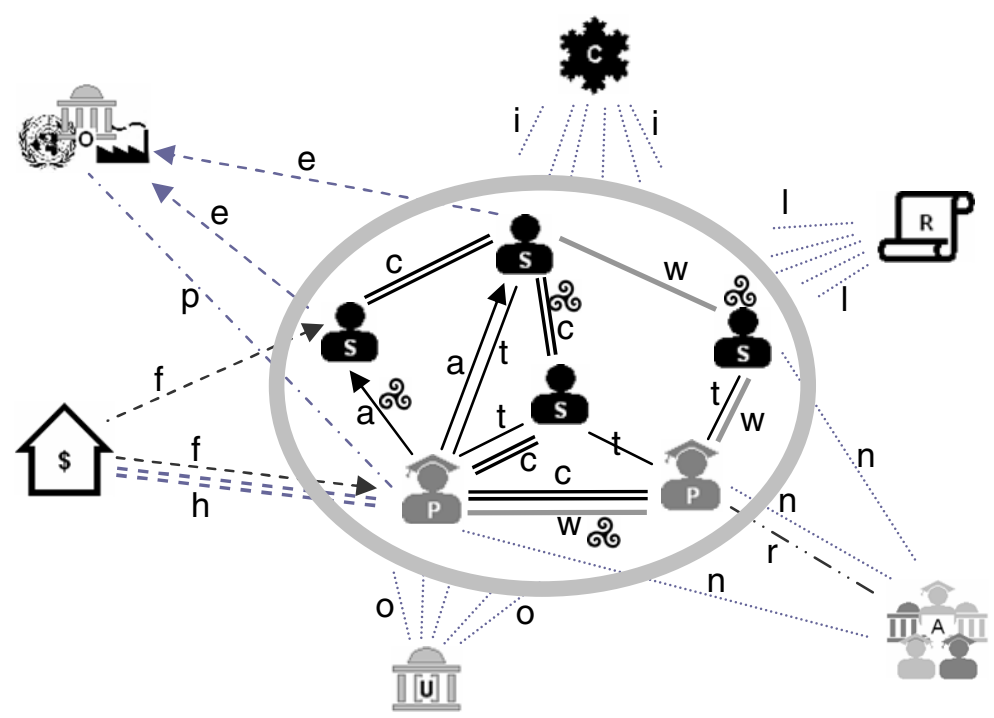

Fig. 1. A CESM diagram for a graduate education system

The endostructure - the bonds between components - are chiefly relations of advising (a), teaching ( $\mathrm{t}$ ), cooperative work in publications and projects $(\mathrm{w})$, and communication (c), including messaging, conversation, collaboration, argumentation, feedforward (coaching), and feedback. The exostructure - the bonds between components and environment - comprise the subjection of all components to law and regulations (l), the reputation (r) and networking (n) relations of some components with the community, organizational ties between all components and the university (o), the funding from agencies to components (f) and their service as experts and ad hoc referees for the agencies (h), partnership (p) and employment (e) relations between components and several organizations, and the cultural influence (i) that flows to and from the academic community.

The mechanism is a process that generates qualitative novelty [4], i.e., a process that drives or blocks its transformations, including the emergence or submergence of the system or some of its properties. As Bunge teaches, most mechanisms are concealed and have to be conjectured. In our current conjecture, we devise four main mechanisms for graduate education excellence, each one represented by a triskelion in Figure 1: ชి

1. Study and research. This seems to be the most important mechanism of all, performed by each student under influence from and communication with adviser, professors, and colleagues.

2. Advising. This establishes a vertical relationship - the student, who has research interests and passions, is supposed to learn from the adviser, who also has interests and passions, but more experience.

3. Cooperative work in publications and projects. This sets a horizontal relationship in which the student is expected to learn from others through the sharing of knowledge and good practice, besides establishing a reputation 
about his competence to deliver research results - something beyond pure cognitive learning.

4. Communication. Not any communication, but the exchange of scientific ideas grounded in strong directives of objective criticism and argumentation, thus establishing a culture, at the collective level of the graduate education system.

Note that, by this choice of mechanisms, we leave out other alternatives such as candidate selection or thesis defense (considering that we don't rely on these processes to 'make the system tick'), or the obtaining of funding (not a mechanism, since funding per se doesn't operate the transformations we look for). Note also that the first mechanism works by transformations at the component level (student), while mechanisms 2 and 3 depend on transformations in small groups of components and their bonds. The fourth mechanism, as we stated it, is a communication culture involving all students and their communication bonds.

The first three mechanisms are object of deliberate action by most if not all graduate programs. The fourth is usually taken for granted like a consequence of individual and small-group actions, a "Hidden Hand" that we little can do about. We decided to deliberately approach the enhancement of scientific argumentation and feedback among the graduate students. This approach is presented next.

\section{Approach to Growing a Peer Review Culture among Students}

Peer review in education has been practiced by the first author of this paper since $1997^{3}$. Kern, Saraiva, and Pacheco [5] discuss its motivation in terms of collaboration, written expression, critical thinking, and professional responsibility. Several other scholars around the world have also practiced it in engineering and computing settings, e.g., [6-14].

Our graduate research seminars started in 2005 as a series of 8 four-hour meetings along the school year, with a peer review of thesis proposals, culminating in a workshop in which all first-year students present their proposals. The seminars are mandatory for first-year students, with no credits or grades. The peer review has been conducted in a double-blind manner, in a single round (although we may experiment with more rounds if funding becomes available). The seminars have the following objectives:

- For the students:

- To know the essential parts of a thesis proposal, then re-elaborate the proposal presented for entrance in the graduate program and participate in a peer review round.

- To develop competence to give and receive professional, objective critique of scientific work.

- For the graduate program:

- To create and disseminate a culture of objective, interdisciplinary scientific criticism.

\footnotetext{
${ }^{3}$ An account of the objectives, publications, and practice is given in "Project PAR: Educational Peer Review", in http://kern.ispeople.org/par_en.html
} 
- To serve as catalyst of the advising process.

- To stimulate interdisciplinary scientific interchange.

In order to fulfill those objectives, students needed individual guidance for writing and critiquing the proposals; otherwise the peer review process would be just an exchange of opinions and uneducated guesses. In our young, multidisciplinary graduate program, the worldviews and methodological approaches among advisers are very heterogeneous (i.e., we are not interdisciplinary yet). The building of an agreement over our interdisciplinary methodological approach is still in its early stage. Therefore we adopted one set of guidelines for the introduction of a proposal that had been successfully in use by the second author of this paper.

That set of guidelines was turned into a template for use by the students to write their proposals, although we decided to omit any methodological aspect since this issue was seen as conflict-prone in a setting of about 40 advisers with varied backgrounds and very little time to work out an agreement that could serve all. The elements of the proposal had minor changes in these 4 years; the basic structure is: Motivation, Problem statement and/or question, Objectives, Relevance, Scope, and Main references.

Another challenge was to choose the software to support the peer review process. Following personal experiences with conference management interfaces and the summaries of Snodgrass ${ }^{4}$ and CommunityWiki ${ }^{5}$, we've found that all conference systems were too ill-adapted for the task. We chose the Open Journal System (OJS ${ }^{6}$ ) for two main reasons: it is a very successful and widespread interface, and it permits attaching a file to the referee report. This allowed us to build a spreadsheet template of a referee report with some fixed parameters and data ranges - for instance, we included in the template self-declarations of (i) expertise in the topic of the proposal (to give a sense of how sure is the critic about specific remarks made), (ii) research area of the referee - Knowledge Engineering (KE), Knowledge Management (KMa), or Knowledge Media (KMe), and (iii) graduate level (doctorate or masters).

Student preparation involved instruction on proposal writing during the first seminars. Additionally, students are required to discuss their proposals with their advisers. Following that, we run a series of seminars (typically three 4-hour sessions) in which about $1 / 3$ of the students have the chance to present and get instant feedback from usually 2 professors (frequently this paper's authors, but with more participants in 2007 and 2008) and occasionally also from colleagues. The feedback focuses on the internal coherence of each proposal's items (e.g., whether the research question is indeed a research question) and on the relational coherence between them (e.g., whether the objectives are compatible with the research question).

The professors who give instant feedback pledge not to judge and not to advise (that's the adviser's work, after all). They only address the form, not the merit of the proposal (because the conditions to engage in a collective, profitable debate on merit

\footnotetext{
${ }^{4}$ Summary of Conference Management Software, by Richard Snodgrass, 1999. http://www.acm.org/sigs/sgb/summary.html

${ }^{5}$ ConferenceManagementSoftware, software to help organize a conference, last edited 200601-18. http://www.communitywiki.org/cw/ConferenceManagementSoftware

${ }^{6}$ Open Journal System, an open source initiative from the Public Knowledge Project from Canadian and American universities. http://pkp.sfu.ca/?q=ojs
} 
are still far from ideal). The discussions are limited to some student proposals, with scarce time, but the goal of these sessions is not to achieve a complete analysis of each proposal. Instead, these sessions should allow students to grasp the principles of objective, rigorous, professional criticism.

With the experience gained in presenting and receiving feedback, or merely in watching colleagues in that situation, the students refine and submit their proposals through OJS. The next step for the students is to give feedback to their colleagues on their thesis proposals. There is instruction on the task of the referee [15]. The first author shows the referee reports for his first actual international submission for illustration, to give the students a sense of the concrete experience of receiving feedback.

The individual thesis proposals are allocated to referees, therefore each student gets also 3 proposals to review. Referee allocation follows two rules: the referees are colleagues at the same graduate level (either doctorate or masters) and each proposal receives a mix of 2 referees from the same area $(\mathrm{KE} / \mathrm{KMa} / \mathrm{KMe})$ and one from a different area.

Two weeks are allowed for the students to read and fill in a referee report for each of the 3 proposals assigned. The anonymous reports (spreadsheets) are collected from OJS and processed in a relational database (MySQL). This allows us to compose a document with all anonymous referee reports and publish it for all participating students and advisers.

\section{Results and Discussion}

\subsection{Results and Opportunities for Reflection}

From 2005 to 2008, we had 49, 67, 54, and 62 student authors, respectively. A few additional students are allowed to take part only as referee, occasionally, allowing for the allocation of 4 referees for some proposals. Table 1 gives the numbers of proposals in 2008, for illustration. Four extra referees took the number of participants to 66 , with 198 referee reports. From those, 192 were delivered.

Table 1. Thesis proposals by research area and by graduate level

\begin{tabular}{r|cc|c}
\hline $\begin{array}{c}\text { Graduate level } \rightarrow \\
\downarrow \text { Research area }\end{array}$ & M.Sc. & Dr. & total \\
\hline Knowledge engineering & 8 & 14 & 22 \\
Knowledge management & 11 & 13 & 24 \\
Knowledge media & 8 & 8 & 16 \\
\hline total & 27 & 35 & 62 \\
\hline
\end{tabular}

Commitment of referees and depth of feedback vary. Although this might be related to a culture of reciprocity in which some students don't put reasonable effort in the refereeing task because they won't get an A, we prefer not to change the "mandatory, no credits, no grade" character of our research seminars. We prefer, instead, to continue working on communication issues that lead to culture 
consolidation - for instance, raising awareness of the importance of the peer review process, giving fuller feedback to student and adviser about timeliness, frequency, and quality of participation of the student in both of his roles.

The general feedback report published for students and advisers right after finishing the review process shows:

- For each proposal

- For each referee report (typically 3 for proposal)

- Research area of the referee

- Referee's self-declaration of expertise

- Grades (0-10) and comments on the topics reviewed

This general report allows each student to:

- See the full referee reports about his proposal, study and compare the reports, and reflect about the quality of his work.

- See details of other referee reports to the same proposals he reviewed, therefore getting information to reflect on his abilities as referee and on the quality of communication bonds (as stated in section 2, a kind of scientific communication guided by principles of objective criticism and argumentation).

The raw data calls for the building of statistics and other summaries and analyses for the comprehension of our graduate learning system. Our approach is a work-inprogress and only recently (2008) we begun to publish aggregates. For instance, data on referee self-declaration of expertise rendered the figures in Table 2. The obvious conclusion - several students don't have an accurate appraisal of their expertise compared to colleagues' - calls for an explanation of why is that so (although common sense points to a culture of false or mandatory modesty). This lack of balance may be evidence of poor communication among the students, who nevertheless spend their first year meeting one another in at least 3 mandatory courses and in the research seminars.

Table 2. Distribution of self-declarations of expertise in 2008

\begin{tabular}{r|cc}
\hline I belong to the third part of students who... & \# reports & \% reports \\
\hline know the most about the proposal's main topic & 28 & 14.6 \\
have average knowledge of the topic & 92 & 47.9 \\
know the least about the proposal's main topic & 71 & 37.0 \\
[did not declare expertise level] & 1 & 0.5 \\
\hline total & 192 & 100.0 \\
\hline
\end{tabular}

Most resources employed in our approach, up until now, were devoted to making the peer review system work smoothly and profitably. We are now ready to take steps in research and development associated to our approach, as discussed next.

\subsection{Opportunities for Improvement and R\&D Issues}

There are instrumental and methodological opportunities to improve our approach. The instrumental issues include, besides providing better computer systems to process 
the bureaucracy of peer review, the automation or semi-automation of several knowledge-intensive tasks. Some of these tasks, as defined by Schreiber et al. [16], are open to a knowledge engineering approach that involves understanding the business context, identifying knowledge assets and knowledge-intensive tasks, taking a strategic decision for task automation, adapting knowledge model templates, and implementing knowledge systems. Some of the candidate tasks for automation are:

- Referee allocation - using, for instance, text mining techniques to match concept clouds from proposals to referees'.

- Rating of referees - for instance, using the approach proposed by Riggs and Wilensky [17].

- Reliability and validity measures (statistics).

- Process evaluation using nonlinear dynamics [18, 19] applied to asynchronous environments - as in the case of a trial by Araújo [20].

There are, also, two wide methodological issues at hand: the articulation of the graduate research seminars with a new mandatory course on the scientific method, and the study of the mechanisms that create emergent properties in our graduate learning system. As for the articulation, our approach has been seriously limited by omitting the assessment of methodological aspects. We need to build an agreed-upon (or at least accepted) set of methodological directions for our theses if we want our program to be interdisciplinary - hence cohesive - instead of multidisciplinary hence potentially dispersive [2].

Up until now, our students and advisers only count on their scientific or technological background for methodological issues. The new course on Method will deepen the understanding of methodological issues and allow for better communication of those aspects. The research seminars will be able, then, to build on that understanding, including the assessment of Method in the reviews.

Regarding the study of a system's mechanisms, we need to evolve from our current mere assumption that the growing of a peer review culture improves our graduate education system (a sort of "Hidden Hand" mechanism hypothesis; only a conjecture) to a proper explanation of how the mechanism works. Bunge [2, 4] has directions for that - for instance, the use of multilevel analysis (micro-macro systems) to uncover the mechanism behind some correlations.

\section{Concluding Remarks}

We presented our approach to grow a peer review culture among graduate students of Knowledge Engineering and Management. The students experience the main scientific method for quality control and have an opportunity to sharpen their knowledge and strengthen their (scientific, rigorous, objective) communication bonds with their peers and professors, adviser included.

The approach is part of our quest for interdisciplinarity through strengthening communication bonds. No single discipline can deal with our research object knowledge as a production factor - and a mere multidisciplinary approach is bound to fail [2]. Our work aims at contributing, as well, to establish peer review as a replicable, scalable educational approach [21]. 


\section{Acknowledgments}

Authors GCS, SR, and RTSL were, at the time of their participation, graduate students who volunteered to perform fundamental tasks associated with the peer review process and its assessment. The authors thank the support provided by Instituto Stela by means of the systems administration services performed by André Ricardo Righetto.

\section{References}

1. Angelov, C., Melnik, R.V.N., Buur, J.: The synergistic integration of mathematics, software engineering, and user-centered design: exploring new trends in education. Fut. Gen. Comp. Sys. 19(8), 1299-1307 (2003)

2. Bunge, M.: Emergence and convergence: Qualitative novelty and the unity of knowledge. University of Toronto Press, Toronto (2003)

3. Bunge, M.: Systemism: the alternative to individualism and holism. J. Socio-Econ. 29(2), 147-157 (2000)

4. Bunge, M.: Mechanism and explanation. Phil. Soc. Sci. 27(4), 410-465 (1997)

5. Kern, V.M., Saraiva, L.M., Pacheco, R.C.S.: Peer review in education: promoting collaboration, written expression, critical thinking, and professional responsibility. Educ. Inf. Technol. 8(1), 37-46 (2003)

6. Hartman, J.: Writing to learn and communicate in a data structures course. In: $20^{\text {th }}$ SIGCSE Technical Symposium on Computer Science Education, pp. 32-36 (1989)

7. Hafen, M.: Developing writing skills in computer science students. ACM SIGCSE Bul. 26(1), 268-270 (1994)

8. Cunningham, S.J.: Using a computer conferencing system to support writing and research skill development. ACM SIGCSE Bul. 26(4), 5-8 (1994)

9. Davies, R., Berrow, T.: An evaluation of the use of computer supported peer review for developing higher-level skills. Comput. Educ. 30(1/2), 111-115 (1998)

10. Gehringer, E.F.: Electronic peer review and peer grading in Computer-Science courses. ACM SIGCSE Bul. 33(1), 139-143 (2001)

11. Liu, E.Z., Lin, S.S.J., Chiu, C., Yuan, S.: Web-based peer review: the learner as both adapter and reviewer. IEEE Trans. Educ. 44(3), 246-251 (2001)

12. Moreira, D.A., Silva, E.Q.: A method to increase student interaction using student groups and peer review over the internet. Educ. Inf. Technol. 8(1), 47-54 (2003)

13. Sitthiworachart, J., Joy, M.: Web-based peer assessment in learning computer programming, In: $3^{\text {rd }}$ IEEE International Conference on Advanced Learning Technologies (ICALT 2003) (2003)

14. Denning, T., Kelly, M., Lindquist, D., Malani, R., Griswold, W.G., Simon, B.: Lightweight preliminary peer review: does in-class peer review make sense? ACM SIGCSE Bul. 39(1), 266-270 (2007)

15. Smith, A.J.: The task of the referee. IEEE Computer 23(4), 46-51 (1990)

16. Schreiber, G., et al.: Knowledge engineering and management: the CommonKADS methodology. MIT Press, Cambridge (2000)

17. Riggs, T., Wilensky, R.: An algorithm for automated rating of reviewers. In: $1^{\text {st }}$ ACM/IEEE-CS joint conference on digital libraries, Roanoke, pp. 381-387 (2001)

18. Losada, M.: The complex dynamics of high performance teams. Math. Comput. Model. 30(9-10), 179-192 (1999) 
19. Losada, M., Heaphy, E.: The role of positivity and connectivity in the performance of business teams: A nonlinear dynamics model. Am. Behav. Scient. 47(6), 740-765 (2004)

20. Araújo, L.H.L.: Uma aplicação da dinâmica não-linear para a avaliação de desempenho de comunidades virtuais de aprendizagem [An application of nonlinear dynamics to performance evaluation of virtual learning communities]. Masters dissertation, Universidade Católica de Brasília, Brasília-DF, Brasil (2004)

21. Kern, V.M., Pacheco, R.C.S., Saraiva, L.M., Pernigotti, J.M.: Peer review in Computer Science education: Requirements for continuous, large scale application. In: Mendes Neto, F.M., Brasileiro, F.V. (eds.) Advances in computer supported learning, pp. 46-65. Idea Group Publishing, Hershey (2007) 